Instituto Internacional de Investigación y Desarrollo Tecnológico Educativo INDTEC, C.A.

DOI: https://doi.org/10.29394/Scientific.issn.2542-2987.2019.4.11.5.103-120

OAI-PMH: http://www.indteca.com/ojs/index.php/Revista Scientific/oai

Artículo Original / Original Article

\title{
Aprovechamiento del Facebook como Herramienta Tecnológica en el Proceso Educativo Ambiental
}

\author{
Autor: Ender Alejandro Morón Hernández \\ Universidad Pedagógica Experimental Libertador, UPEL \\ arbolmoron@gmail.com \\ Barinas, Venezuela \\ https://orcid.org/0000-0001-6697-9109
}

Resumen

La presente investigación tiene como objetivo proponer el aprovechamiento del Facebook como herramienta tecnológica en el proceso educativo, en la ETAS "San José" Barinas. La presente investigación se desarrolló bajo la modalidad de Proyecto Factible, fundamentada en una investigación de campo de carácter descriptivo; con una población de 38 sujetos conformados por Director, Coordinadores, Técnicos de Campos y Docentes de áreas. Para la selección de la muestra por ser pequeña y representativa se tomó la totalidad de la población. Para la recolección de la información se empleó la técnica de la encuesta y como instrumento se utilizó un cuestionario de 20 ítems de preguntas cerradas, con escala de Likert (Siempre, Casi Siempre, Algunas Veces, Nunca). La validez del instrumento se realizó a través de la técnica juicio de expertos. Para la obtención de confiabilidad se aplicó el coeficiente Alpha de Cronbach arrojando resultados de 0,90 En la investigación planteada, la propuesta está dirigida a los docentes para el uso de dicha herramienta tecnológica en el proceso de enseñanza y aprendizaje. De igual manera, incluir dentro de la planificación contenidos relacionados al medio ambiente y diseñar estrategias que logre despertar el interés y la motivación en los estudiantes.

Palabras clave: red social; proceso de aprendizaje; educación ambiental. 


\title{
Use of the Facebook as a Technological Tool in the Environmental Educational Process
}

\begin{abstract}
The objective of this research is to propose the use of Facebook as a technological tool in the educational process, at the "San José" Barinas ETAS. The present investigation was developed under the modality of Project Feasible, based on a field investigation of descriptive character; with a population of 38 subjects made up of Director, Coordinators, Field Technicians and Teachers of areas. For the selection of the sample because it is small and representative, the entire population was taken. For the collection of information, the survey technique was used and a questionnaire of 20 items of closed questions was used as a tool, with a Likert scale (Always, Almost Always, Sometimes, Never). The validity of the instrument was carried out through the expert judgment technique. In order to obtain reliability, Cronbach's Alpha coefficient was applied, yielding results of 0.90 . In the proposed research, the proposal is aimed at teachers for the use of said technological tool in the teaching and learning process. Likewise, include within the planning content related to the environment and design strategies that will arouse interest and motivation in students.
\end{abstract}

Keywords: social network; learning processes; environmental
ation. education.

Date Received: 12-03-2018

Date Acceptance: 20-08-2018 


\section{Introducción}

La humanidad ha experimentado distintas formas de supervivencia, producto de su evolución que la impulsa constantemente a la búsqueda de alternativas para satisfacer sus expectativas de vida. Entre estas se tiene la protección del ambiente, pues este implica bienestar en el más amplio sentido de la existencia y en su afán de conseguirlo procura interactuar con el entorno, para ello alterna con las nuevas innovaciones tecnológicas, tal es el caso de la red social Facebook, donde ésta hoy día tiene un alto índice de usuarios, de allí la oportunidad para su aprovechamiento en el buen sentido de la palabra, es decir por medio de esta se puede lograr minimizar la amenaza en la que se ha visto sometida el planeta en los últimos años por la contaminación ambiental.

Actualmente la revolución digital lleva a las empresas a comprender la importancia de innovar y que no pasen al olvido. Mejorar las plataformas de servicio y adaptarse a las nuevas necesidades de los usuarios ultra conectados ya no es una opción, de hecho, se ha convertido en algo crucial para mantenerse competitivo en el mercado que es cada vez más virtual y menos físico.

De igual manera, la tecnología evolucionando de forma rápida junto con la comunicación entre los consumidores y las plataformas de negocio, ante la diversificación de los canales de comunicación y las nuevas expectativas de los usuarios, quienes ahora tienen acceso a diferentes dispositivos e innovaciones. Esta tendencia ha provocado que las organizaciones reconozcan la experiencia de las instituciones $y / 0$ empresas como un diferenciador clave para conquistar al usuario moderno. Por otro lado, se debe tener en cuenta, que las redes sociales figuran como los principales canales que generan los datos de mayor valor para innovar, donde el manejo adecuado de esta información dependerá de contar con una plataforma calificada para impulsar el crecimiento exponencial y de esta manera, asegurar su 
supervivencia en la continua revolución tecnológica.

Bajo esta premisa, emerge la necesidad de sumar voluntades haciendo uso de los distintos medios sociales con el fin de contrarrestar el daño irreparable que sufre el ambiente, En tal sentido, el presente estudio se aborda para contribuir de forma inmediata a la mencionada problemática, por medio del uso adecuado a las redes sociales, el cual favorece el proceso educativo. La humanidad ha experimentado distintas formas de supervivencia, producto de su evolución que la impulsa constantemente a la búsqueda de alternativas para satisfacer sus expectativas de vida. Entre estas se tiene la protección del ambiente, pues este implica bienestar en el más amplio sentido de la existencia y en su afán de conseguirlo procura interactuar con el entorno, para ello alterna con las nuevas innovaciones tecnológicas, tal es el caso de la red social Facebook, donde ésta hoy día tiene un alto índice de usuarios, de allí la oportunidad para su aprovechamiento en el buen sentido de la palabra, es decir por medio de esta se puede lograr minimizar la amenaza en la que se ha visto sometida el planeta en los últimos años por la contaminación ambiental.

Al respecto, existe la necesidad de proteger la naturaleza, a través de la concienciación de la especie humana para el saneamiento del ambiente, pues es fundamental para la vida, por ello surge la inquietud de realizar múltiples investigaciones con el propósito de encontrar soluciones sostenibles a cada uno de los grandes problemas generados por la contaminación ambiental y de esta manera emprender acciones para retribuir a la naturaleza el daño que se le ocasiona. Se requiere la participación activa del proceso educativo en la concienciación ambiental en todos sus ámbitos, niveles, sistemas y modalidades así como en los grupos sociales, para la transformación del comportamiento asumido por el ser humano en cuanto al daño ambiental se refiere, de ahí la necesidad de la presencia en la educación ambiental en el quehacer diario, para que surta efecto multiplicador, no sólo bajo la responsabilidad de los centros educativos, sino con el trabajo 
mancomunado de los actores sociales, pues mantener el ambiente en equilibrio es competencia de todos los ciudadanos, sin embargo este hecho social parece ser indiferente para muchos, de acuerdo a publicación editada por VITALIS (2013), señala lo siguiente:

Pocos esfuerzos en materia educativa ambiental, que formen en valores a la ciudadanía y promueva nuevas actitudes en favor de un ambiente sano y ecológicamente equilibrado. Muchos de los esfuerzos desarrollados en el período evaluado se articulan más en campañas publicitarias que educativas, olvidando la necesidad de permanecer en el tiempo para lograr los cambios de conducta necesarios en la materia, a los fines de abordar y resolver los principales problemas ambientales que afectan al país (pág. 9).

En tal sentido, la participación integradora de la ciudadanía es indispensable desde dos vertientes: a). la primera, promoviendo información sobre la educación ambiental con herramientas o estrategias de gran alcance para enfrentar el desafío ambiental y; b). la segunda, asumir el compromiso de dicha información que impliquen cambio o transformación de conductas con las acciones en función del equilibrio ambiental.

En consecuencia, se requiere de manera activa y en todos los ámbitos darle el lugar que merece la educación ambiental en el proceso educativo para dar respuesta a dicha problemática, por ello el aprovechamiento de la red social Facebook como herramienta tecnológica, la cual ha tomado gran importancia en la sociedad, donde además debe instituirse como aliada en los centros educativos, específicamente en la Escuela Técnica Agronómica Salesiana "San José" del estado Barinas, ya que ésta en su filosofía institucional facilita la comunicación y el aprendizaje colaborativo e introduce nuevas formas de trabajo entre los actores del proceso educativo como parte de la formación integral. Así pues, lo medios de interacción comunicacional llamados redes sociales, de acuerdo con Cabrera (2014): quien señala en un estudio realizado por comScore Media Metrix, menciona lo siguiente "La 
penetración de las redes sociales entre los usuarios de internet es alta en Venezuela, en el 90,2\% de las personas de 15 años o más" (pág. 87). La intención plena es idear la forma en que esta herramienta tecnológica sirva de apoyo al proceso educativo ambiental, es decir, que haya un requerimiento de espacios educativos y formativos por parte de los facilitadores de la ETA "San José", en el uso del Facebook.

De allí, la razón de ser de dicha institución se fundamenta en el área agrícola, comprometida con el quehacer ambiental, y no existen políticas o acuerdos a nivel directivo donde haya aunque sea una (1) asignatura por año escolar vinculada al ambiente, contempladas en el diseño curricular con contenidos para que el docente y el estudiante intercambien espacios ambientales. Lo anterior expuesto, demuestra la ausencia de espacios tecnológicos (red social Facebook) que debe tener el docente como estrategia didáctica ambiental en su proceso de formación académica, en aras de buscar soluciones a la problemática ambiental que se vive a diario.

Por otro lado, se tiene la explicación sobre las redes sociales de Ojeda, Gutiérrez y Perales (2009): "también puede promover una vertiente de sensibilización ambiental y de aportación en campañas de diferente índole" (pág. 320). Es indudable, que las innovaciones tecnológicas han sido pieza clave e indispensable en cualquier contexto, llámese educativo, laboral, organizacional y gubernamental, tanto es así, que se han diseñados programas, proyectos y jornadas a nivel de la Educación Ambiental a nivel mundial, donde a través de diversas campañas de sensibilización y concienciación se promueven trabajos de recuperación de los espacios ambientales. Por otro lado, se difunden por medio de redes sociales, lo cual es una manera de informar y dar a conocer cada proyecto ambientalista que se esté ejecutando y entre otras, permite intercambiar ideas, opiniones y pensamientos sobre la educación ambiental, dentro de este ámbito la participación de la población estudiantil, es atrayente por sus habilidades para 
ingresar a las redes sociales.

En este sentido, la problemática suscitada en la ETA "San José" se puede abordar desde la actitud desinteresada, falta de creatividad o iniciativa por parte de los docentes de la institución en darle uso a la red social Facebook o crear espacios relativamente sencillos que sirvan de enlace entre los actores del proceso educativo ambiental, para que haya una integración efectiva y dinámica en cuanto a la amenaza ambiental existente en el mundo, es buscar la forma de difundir campañas en defensa del ambiente a modo de hacer partícipe a todos los ciudadanos con acciones que se requieren para hallar el equilibrio entre hombre y ambiente. Así lo señala Tobasura (2006): "la gente recuerda mejor lo que hace, y para ello se requiere la participación activa" (pág. 398). En consecuencia, surge la siguiente interrogante: ¿Cómo los docentes pueden aprovechar adecuadamente las innovaciones tecnológicas como una herramienta en el proceso de formación ambiental en la Escuela Técnica Agronómica Salesiana "San José" del estado Barinas? De igual forma se plantea el siguiente propósito: promover el aprovechamiento de herramientas tecnológicas para el proceso educativo ambiental en la Escuela Técnica Agronómica Salesiana "San José" Barinas estado Barinas

\section{Redes Sociales}

En este apartado, se analiza la variable de la red social Facebook es necesario hacer mención de lo general a lo específico, es decir, comentar a groso modo acerca de las redes sociales como elemento de comunicación social, la cual su finalidad es la interacción entre los individuos de la sociedad, quienes hacen uso de distintas redes para comunicarse. Por tanto, Orihuela (2008a), explica claramente:

Las redes sociales en línea, como Linkedln, Facebook o Tuenti, son servicios basados en la web que permiten a sus usuarios relacionarse, compartir información, coordinar acciones y en general, mantenerse en contacto. Estas aplicaciones son la 
nueva forma en la que se representa nuestra red social, pero también son el modo en el que se construye nuestra identidad online y el cauce mediante el que se agrega y comparte nuestra actividad en la red (pág. 58).

En atención a lo expuesto, la invención de dichas redes contribuye a la interacción del hombre, es decir, pasan de un simple ordenador que solo servía para dejar información a convertirse en un intercambio de ideas, opiniones y sentimientos aunado a los intereses de los usuarios; la evolución progresiva de estas innovaciones tecnológicas permite desde el punto de vista pedagógico nuevas posibilidades en el aprendizaje conducidos al quehacer educativo y formativo del ser humano.

Por ello, la aparición de las redes sociales, más la inserción de estas en el campo educativo puede apreciarse como una de las herramientas tecnológicas eficaz para ser aprovechada al máximo en los aprendizajes de la vida, de allí pues la aplicación de dicha herramienta en el proceso educativo ambiental de la ETAS "San José de Barinas, por medio de la creación de espacios o ambientes que fomenten educación y formación en el área ambiental convertirá a la institución antes mencionada en un centro educativo que aprende. En definitiva, Orihuela (2008b), describe las redes sociales así: "las redes sociales son los nuevos espacios virtuales en los que nos relacionamos y en los que construimos nuestra identidad" (pág. 59).

En concordancia, con el autor quien expresa claramente que las redes sociales sirven para que exista comunicación entre grupos según su interés o necesidad, por ello al hacer uso de las redes sociales particularmente del Facebook, según lo referido en la investigación, la cual busca el fortalecimiento de la educación ambiental por medio del proceso educativo, como tema de interés que le concierne al mundo entero. 


\subsection{Tipos de Redes Sociales}

Actualmente, se observa un amplio perfeccionamiento vinculado en las innovaciones tecnológicas, debido al avance que han venido desarrollando en el tiempo y el espacio. De allí la importancia de las redes sociales en la sociedad actual, reflejando la necesidad del ser humano de expresión y reconocimiento, de acuerdo con la necesidad, en fin, todas las que permitan interacción con el entorno. De acuerdo con Boyd y Ellison (2007), citados en Flores, Morán y Rodríguez, (2009), realizó un artículo sobre redes sociales señalado así:

Una red social se define como un servicio que permite a los individuos (1) construir un perfil público o semipúblico dentro de un sistema delimitado, (2) articular una lista de otros usuarios con los que comparten una conexión, y (3) ver y recorrer su lista de las conexiones y de las realizadas por otros dentro del sistema. La naturaleza y la nomenclatura de estas conexiones pueden variar de un sitio a otro (pág. 3).

Es innegable, que los avances en el tema de las innovaciones tecnológica ha venido llenando y ganando espacios hoy en día, las redes sociales tienen ya acaparados todos los espacios en el mundo global, la cibernética ya es indispensable en el ser humano o mejor dicho en la sociedad mundial, allí se expresa, la necesidad de estar a tono, capacitado, abierto a los cambios tecnológicos especialmente en las redes sociales específicamente el Facebook, que ya tiene en el mercado toda la audiencias atenta a cambios y nuevas herramientas de innovaciones tecnológicas.

En este orden de ideas, la mencionada red social, permite desde una perspectiva tecnológica y alcance educativo en el área ambiental una forma de interactuar en cualquier contexto, empresarial o con fines educativo, es decir, ahondar sobre el beneficio que se puede tener con el Facebook como red social, la cual tiene un alto índice de usuarios como posibilidades de crear información que implica la formación académica en el proceso educativo. Bajo 
esta premisa, la formación ambiental dirigida a los estudiantes de la ETAS "San José" de Barinas, ya que los mismos se adaptan con facilidad al medio comunicacional masivo que ofrece la tecnología para crear y difundir mensajes educativos ambientales dirigido al contexto educativo orientados $y$ supervisados por los docentes de la institución educativa antes mencionada.

En este mismo contexto, la presente investigación es innovador, ya que permite a los usuarios de la red una herramienta académica que puede ser utilizada desde diversos contextos y ámbitos, siendo aprovechado efectivamente para lograr la comunicación asertiva con el mundo que lo circunda. Por otro lado, se fundamenta en el intercambio de conocimientos, asentando las oportunidades como herramienta tecnológica ofrecidas por medio del ámbito educativo. De esta manera, se debe asumir el compromiso que involucra el aprendizaje en la formación académica, pues no sólo los estudiantes obtendrán provecho en el uso del Facebook, sino todos los actores involucrados en este proceso, ya que la temática a desarrollar busca la interacción hombre-ambiente.

La puesta en marcha de un espacio en Facebook, donde se cumpla con los procesos administrativos, considerando esta herramienta para la educación y formación con estrategias dinámicas, constructiva, permitirá un cambio de conducta en aquellas personas con actitudes negativas ante el sistema ambiental, también genera valores ambientalistas, donde los usuarios sean capaces de crear sus propios espacios en la web a fin de transferir esas capacidades por medio de sus experiencias, fortalecidas en el conocimiento obtenido en el intercambio comunicacional.

Al respecto, la cuenta Facebook es de fácil acceso incluso nada complejo para crearla; se hace referencia a ello, ya que permite observar el abanico de oportunidades que se tiene para crear espacios de educación ambiental, es decir si estos lugares son visitados por una cantidad de usuarios de acuerdo a sus necesidades, entonces el proceso educativo en esta red 
social merece el mejor uso y aprovechamiento y con mayor efectividad el tema de saneamiento ambiental de manera específica a nivel nacional, regional y local como empuje al proceso educativo en la ETAS "San José" de Barinas.

Con respecto, a esta premisa en la era digital, lo que se quiere es crear un auténtico cambio de civilización; de acuerdo con un Congreso Celebrado en Granada en el año 2004. Sevillano (2008): afirma que "serían competencias exigibles a todos los docentes, las vinculadas a las TIC, ya que los nuevos saberes relacionados con las tecnologías presentan en la actualidad nuevos contenidos educativos que poseen un carácter instrumental, procedimental y profesionalizador" (pág. 12). Así pues, la sociedad actual brinda relevancia a la educación, convencida de la recompensa brindada tanto el bienestar individual como colectivo. Actualmente las redes sociales tienen un gran impacto en la vida de los estudiantes, por tal razón los profesores se enfrentan día a día ante la disyuntiva de utilizarlas o no como una herramienta didáctica más, con el objetivo de acercarlos a la realidad, ya que es innegable que el uso de las redes sociales constituyen una parte importante en su vida cotidiana, por lo que es necesario garantizar a los estudiantes involucrarse a los nuevos cambio tecnológicos para que sean capaces de desarrollar un espíritu crítico de los contenidos que producen y emiten.

\subsection{Educación Ambiental}

En otro orden de ideas, se expone la educación ambiental, la cual, representa una alternativa para extinguir el grave problema a nivel mundial, nacional, regional y local, que implica la contaminación ambiental, ésta permite el rescate de valores y cultura para la transformación de la conducta negativa adquirida por los ciudadanos, por tanto, es una herramienta que contribuye a darle el mejor uso a los recursos ambientales. Al respecto, la (UPEL, 2000): señala a la educación ambiental como "un proceso dirigido a mejorar las relaciones ecológicas entre el ser humano y la naturaleza y las relaciones entre 
los individuos, con sentido de compromiso con las generaciones futuras" (pág. 19).

Con respecto, la cita hace referencia precisamente al bienestar que obtienen los individuos por su comportamiento armónico con la naturaleza, conociendo que la permanencia en el planeta no es un hecho aislado, sino más bien la suma de esfuerzos y compromiso asumidos por el hombre-mujer en cuanto a la forma en que debe ser tratado el ambiente. La sociedad, debe asumir con responsabilidad las acciones correspondientes al bienestar ambiental, por ello, es válido echar mano a los recursos disponibles en concordancia con el proceso educativo, como sistema social al alcance de todos. En consecuencia, la expansión de posibilidades que brinden apoyo para el cuidado ambiental, representan la buena intención de los actores sociales, por tanto los aportes realizados son válidos considerarlo en función de acciones ambientales, tal es el caso de la inserción del Facebook en el proceso educativo ambiental en la ETAS "San José" del estado Barinas, a fin de obtener una participación masiva y efectiva de los miembros de dicha institución y por ende, beneficios ambientales.

\section{Metodología}

La metodología utilizada pretende proponer el aprovechamiento de la red social Facebook para la educación ambiental como herramienta tecnológica en el proceso educativo de la ETAS "San José" de Barinas de conformidad a este tópico central se plantean el tipo de métodos y procedimientos técnicos, en cuanto a la naturaleza de la investigación, está enmarcada desde el punto de vista cuantitativo, señalada por Hurtado y Toro (2001): la Investigación Cuantitativa "tiene una concepción lineal, es decir, que haya claridad entre los elementos que conforman el problema, que tenga definición, limitarlos y saber con exactitud donde se inicia el problema" (pág. 76). Por otra parte, este estudio se enmarca, en una investigación proyectiva, 
según Hurtado (2006), se ocupa de: "como deberían ser las cosas para alcanzar unos fines y funcionar adecuadamente" (pág. 325). También se orienta con diseño de campo que para Arias (2006): la investigación de campo "consiste en la recolección de datos directamente de los sujetos investigados o de la realidad donde ocurren los hechos, sin manipular o controlar variable alguna..." (pág. 31); Asimismo, la investigación se ubica dentro de la modalidad de Proyecto Factible, apoyado en una investigación de campo de carácter descriptivo y documental. Es un Proyecto Factible, por cuanto el objetivo es proponer el aprovechamiento de la red social Facebook para la Educación Ambiental como una herramienta tecnológica en el proceso educativo de la ETAS "San José" del estado Barinas. Los proyectos factibles permiten una descripción de los hechos o situaciones de la realidad objeto de estudio, realidad esta que despierta el interés del investigador; 'definido este por la (UPEL, 2012a): "Un proyecto factible consiste en la investigación, elaboración y desarrollo de la propuesta de un modelo operativo viable para solucionar problemas, requerimientos o necesidades de organizaciones 0 grupos" (pág. 21).

También, el proyecto factible comprende las siguientes etapas generales (UPEL, 2012b):

Diagnóstico, planteamiento y fundamentación teórica de la propuesta; procedimiento metodológico, actividades y recursos necesarios para su ejecución; análisis y conclusiones sobre la viabilidad y realización del Proyecto; y en caso de su desarrollo, la ejecución de la propuesta y la evaluación tanto del proceso como de sus resultados (pág. 21).

De la cita planteada, se obtiene que el proyecto factible permite investigar y desarrollar mediante un modelo operativo resolver el problema trazado y aportar soluciones a las necesidades encontradas, que a continuación se describen:

Fase I. Diagnóstico: se indaga a través de la observación, para 
comprobar el planteamiento del problema el cual consiste en el desaprovechamiento de la red social Facebook para la educación ambiental como una herramienta tecnológica en el proceso educativo de la ETAS "San José" del estado Barinas. Asimismo, se fundamenta en teorías para llegar al procedimiento metodológico, el cual se aplicó un cuestionario al personal que labora en dicha institución.

Fase II. Factibilidad: en vista de que el presente estudio estará fundamentado en un proyecto factible, se requiere establecer la factibilidad de este, para la viabilidad científica del proyecto. En esta segunda fase en primer lugar consiste en determinar el estudio de factibilidad tomando en cuenta los aspectos como: social, económico y técnico. Con relación al aspecto social a que sujetos van dirigido el estudio, mientras, lo económico se determina mediante la realización de un presupuesto donde se exponen los costos del material. En cuanto a lo técnico son los recursos necesarios ya sean humanos, financieros, materiales, equipos, entre otros. Por otra parte, la planificación, organización, selección de actividades y estrategias que deberá realizar el investigador con el objeto de prever los recursos humanos, técnicos, materiales y equipos, además planificar las etapas requeridas para la aplicabilidad de la propuesta.

Fase III. Diseño de la Propuesta: Finalmente, en la última fase y atendiendo los resultados del diagnóstico con respecto a la problemática planteada se requiere idear una propuesta como alternativa en cuanto, al aprovechamiento de la red social Facebook como herramienta tecnológica orientada a resultados en los procesos de enseñanza y aprendizaje inmersos en el hecho educativo. 
Cuadro nro. 1. ¿Usa la red social Facebook como herramienta tecnológica en el proceso educativo?

\begin{tabular}{|l|c|c|}
\hline \multicolumn{1}{|c|}{ Criterios } & F(a) & F (\%) \\
\hline Siempre & 0 & 0 \\
\hline Casi Siempre & 0 & 0 \\
\hline Algunas Veces & 10 & 26 \\
\hline Nunca & 28 & 77 \\
\hline Total & 38 & 100 \\
\hline
\end{tabular}

Fuente: Datos obtenidos del cuestionario aplicado; El Autor (2018).

Cuadro nro. 2. ¿Considera que la red social Facebook puede usarse como herramienta aplicable a la defensa y custodia del ambiente?

\begin{tabular}{|l|c|c|}
\hline \multicolumn{1}{|c|}{ Criterios } & F(a) & F (\%) \\
\hline Siempre & 3 & 8 \\
\hline Casi Siempre & 25 & 66 \\
\hline Algunas Veces & 10 & 26 \\
\hline Nunca & 0 & 0 \\
\hline Total & 38 & 100 \\
\hline
\end{tabular}

Fuente: Datos obtenidos del cuestionario aplicado; El Autor (2018).

\section{Conclusiones}

Se deduce de los resultados obtenidos, que los docentes de la ETAS "San José" Barinas, como se expone en el cuadro nro. 1, en su totalidad no hacen uso de la red social Facebook para impartir el proceso de enseñanza aprendizaje inmerso en el quehacer educativo, pues los mismos consideran que los estudiantes le restan importancia al aprendizaje a través de las redes, que si bien es cierto son utilizadas para fines personales, como chatear, subir fotografías, interacción personal, entre otros, dejando a un lado el fin educativo, sin embargo enfatizan la relevancia de darle uso a las herramientas tecnológicas por medio de las redes sociales para el intercambio de saberes el cual incide en un aprendizaje significativo. Por otro lado, se observa en el cuadro nro. 2, que el más alto índice de repuesta se centra en el criterio "casi 
siempre" que representa un resultado favorable para el uso de la red social lo que indica valedera la propuesta orientada a promover y difundir programas ambientales en el proceso educativo en los estudiantes de la ETAS "San José" Barinas. En tal sentido, es necesario recomendar la puesta en práctica de la anteriormente mencionada red social para generar actitudes educativas y formativas en los estudiantes en cuanto a los programas ambientales, con orientación de los docentes.

\section{Referencias}

Arias, F. (2006). El Proyecto de investigación. Introducción a la Metodología Científica. 5ta Ed. Caracas, Venezuela: Editorial Episteme, C.A.

Boyd, D., \& Ellison, N. (2007). Social network sites: Definition, history, and scholarship. Journal of Computer-Mediated Communication, 13(1), 210-230, article 11, ISSN: 1083-6101. Recuperado de:

https://doi.org/10.1111/j.1083-6101.2007.00393.x

Cabrera, M. (2014). En Venezuela, los usuarios de Facebook son mayoritariamente jóvenes. Caracas, Venezuela: Instituto Internet. Recuperado de:

http://blog.institutointernet.net/en-venezuela-los-usuarios-de-facebookson-mayoritariamente-jovenes/

Flores, J., Morán, J., \& Rodríguez, J. (2009). Las redes Sociales. Enlace Virtual, (1), 1-15. Recuperado de:

http://mc142.uib.es:8080/rid\%3D1HY8TVCBB-15599LW1S6Z/redes sociales.pdf

Hurtado, I., \& Toro, G. (2001). Paradigmas y Métodos de Investigación en Tiempos de Cambio. 4ta ed. Valencia, Venezuela: Episteme. Hurtado, J. (2006). El Proyecto de Investigación. Metodología de la 
Investigación Holística. 4ta Ed. Caracas, Venezuela: Ediciones Quirón-Sypal.

Ojeda, F., Gutiérrez, J., \& Perales, J. (2009). ¿Qué Herramientas Proporcionan las Tecnologías de la Información y la Comunicación a la Educación Ambiental?. Revista Eureka sobre Enseñanza y Divulgación de las Ciencias, 6(3), 318-344, ISSN: 1697-011X. Recuperado de:

https://revistas.uca.es/index.php/eureka/article/view/3677

Orihuela, J. (2008a,b). "Internet, la hora de las redes sociales". Nueva revista, (119), 57-62. Recuperado de:

http://www.ecuaderno.com/2008/10/14/la-hora-de-las-redes-sociales/

Sevillano, M. (2008). Nuevas Tecnologías en educación Social. Aravaca, Madrid, España: Editorial McGraw-Hill/Interamericana de España, S.A.

Tobasura, I. (2006). Ambientalismos y ambientalistas. El ambientalismo criollo a finales del siglo XX. 1ra Edición. Manizales, Colombia: Universidad de Caldas.

UPEL (2012a,b). Manual de Trabajos de Grado de Especialización y Maestría y Tesis Doctorales. 4a Ed. Caracas, Venezuela: Fondo Editorial de la Universidad Pedagógica Experimental Libertador FEDUPEL.

UPEL (2000). Educación Ambiental. Caracas, Venezuela: Fondo Editorial de la Universidad Pedagógica Experimental Libertador FEDUPEL.

VITALIS (2013). Situación Ambiental de Venezuela 2012. Análisis de Percepción del Sector. Editores y Compiladores: D. Díaz Martín, Y. Frontado, M. Da Silva, A. Lizaraz, I. Lameda, V. Valera, C. Gómez, E. Monroy, Z. Martinez, J. Apostólico y G. Suárez. 42 págs. [Documento en línea]. Recuperado de:

http://www.ucv.ve/fileadmin/user upload/cenamb/Situacion-Ambientalde-Venezuela-2012.pdf 


\section{Ender Alejandro Morón Hernández \\ e-mail: arbolmoron@gmail.com}

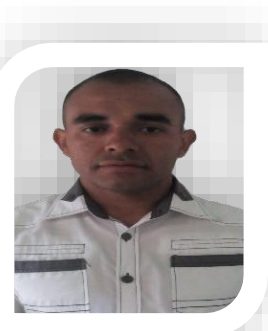

Nacido en Boconó, estado Trujillo, Venezuela. Licenciado en Educación Mención Docencia Agropecuaria en la Universidad Experimental Simón Rodríguez, Núcleo Araure, estado Portuguesa (2011); Técnico Superior Universitario en Tecnología Agrícola Mención Fitotecnia en la Fundación La Salle de Ciencias Naturales, IUTEMAR, Extensión Boconó. Actualmente me desempeño como Técnico de Campo en la Escuela Técnica Agronómica Salesiana "San José" Barinas, estado Barinas, desde el año 2007. Facilitador en el área de Informática en la Escuela Estadal Cerro de Cunaviche, Parroquia Guaramacal, abril-junio 2007. Municipio Boconó, estado Trujillo. 